\title{
Communication on Requirements Elicitation and Interaction Design through SPIDe
}

\author{
Jean C. S. Rosa ${ }^{\alpha, \beta}$, Beatriz B. do Rêgo ${ }^{\alpha}$, Filipe A. Garrido ${ }^{\alpha}$, \\ Pedro D. Valente ${ }^{\beta, \gamma}$, Nuno Nunes ${ }^{\beta, \delta}$, and Ecivaldo Matos ${ }^{\alpha}$ \\ ${ }^{\alpha}$ Department of Computer Science, Federal University of Bahia - UFBA, Salvador, Brazil \\ ${ }^{\beta}$ ITI/LARSYS, M-ITI, Funchal, Portugal \\ ${ }^{\gamma}$ University of Madeira - UMa, Funchal, Portugal \\ ${ }^{\delta}$ Tecnico, University of Lisbon - ULisbon, Lisboa, Portugal
}

\{jean.rosa, beatrizbr, filipe.garrido\}@ufba.br, pvalente@uma.pt, nunojnunes@me.com, and ecivaldo@ufba.br

\begin{abstract}
Participatory Design has a large number of techniques that can be used for requirements elicitation and interaction design. However, choosing a technique (or set of them) suitable for both processes can be challenging. In this sense, in this paper, we present a semio-participatory methodological process - SPIDe - for requirements elicitation integrated with interaction design, by means of a case study, with the objective of investigate if the results of SPIDe application satisfies the needs and desires of users, and how communication process occurs during SPIDe application. This paper contributes to the use of Semiotics and Participatory Design for requirements engineering and interaction design, to the integration of both areas, and to the SPIDe application.
\end{abstract}

Index Terms-User participation, human factors, Semiotic Engineering

\section{INTRODUCTION}

Several human factors influence software conception and development. Some of these factors are research topics in Software Engineering and Human-Computer Interaction (HCI), as for example gender, ethnicity, personality, culture, and social environment [2]. Communication is also one of these factors [2], and one of the current challenges of Requirements Engineering (RE).

Communication is a cultural process and is a study object of Semiotics, which is realized through signs [3]. De Souza [3] argues that the user interface (UI) is a channel of communication in human-computer interaction. But for the UI conception it is necessary to know users' ${ }^{1}$ needs, desires, and constraints for a solution, in order words, it is necessary to know the requirements.

A requirement is therefore an attribute which is defined in order to solve a given problem according the users' needs, desires, and constraints [1]. For requirements to be elicited, there must be communication between users and requirements

DOI reference number: 10.18293/SEKE2019-200

${ }^{1}$ We assume as "problem owners", the customers, subject-interested, (potential) end users, or stakeholders. The term user will always indicate this group. engineers $^{2}$. Communication is the key of RE and must be effective [4]. However, for this effectiveness to take place, it is not enough to simply put the engineer in contact with users, but also to use techniques that facilitate communication [4].

From this perspective, Rosa and Matos [13] consider that the users' effective participation, in the role of design partners, can facilitate communication between designers, engineers, and users, during requirements elicitation and interaction design, which in turn can also facilitate interaction/communication between users and software.

In this paper, we present SPIDe [10]-[13] (a semioparticipatory methodological process), and an investigation about if the results of SPIDe application satisfies the needs and desires of users, and how communication process occurs during SPIDe application. For this, a case study was carried out and the data was analyzed using open and axial codings of Grounded Theory. This paper contributes to the use of Semiotic and Participatory Design to integrate requirement elicitation and interaction design.

This paper is organized in 5 sections as follows: SPIDe is presented and detailed in the next section. In turn, the research methodology is described in Section 3; the research results and the conclusions are presented in Sections 4 and 5, respectively.

\section{SPIDE}

To facilitate the communication between the designer and users through the UI, Rosa and Matos [13] suggest that users become interaction co-authors using the semio-participatory process. Thus, Rosa and Matos [13] developed a process for interaction (re)design called SPIDe. SPIDe is (currently) composed of five PD techniques (contextual inquiry, storytelling, brainstorming, braindraw, and think-aloud) and is based on communication-centered design (CCD) [10], as shown in Figure 1. CCD is an interaction design practice based on Semiotic Engineering [13]. Starting from the interaction concept as a communication between the designer and the

\footnotetext{
${ }^{2}$ In this paper, when considering the engineer, we take into account subjects that practice the Requirements Engineering and all other professionals who work in the software conception and development, such as system developers and analysts.
} 
users through the UI (as defined by Semiotic Engineering) [3], the interaction design for the CCD is a process of messages construction/manipulation sent from designers to users [13].

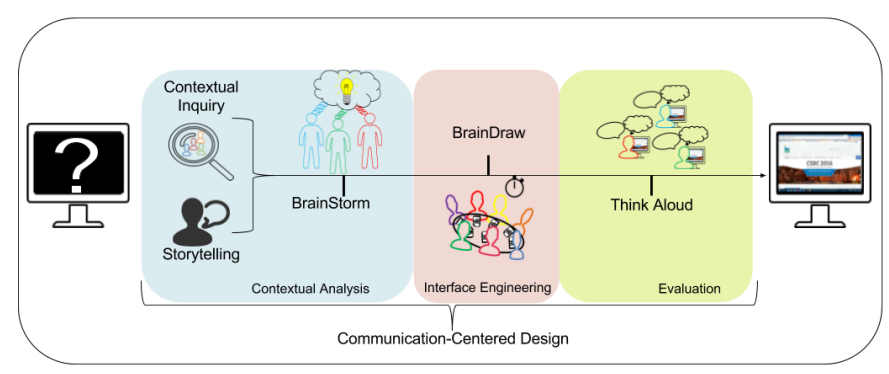

Fig. 1. SPIDe process [10], [13].

\section{1st stage: Contextual Analysis}

The first stage is the contextual analysis, which has three PD techniques: contextual inquiry, storytelling, and brainstorm. The contextual analysis stage aims to get to know the users, identify their contexts, characteristics, problems, desires, needs and understand how it is possible to resolve their problem. In addition, it is at this point that the engineer must understand the impact that the solution will produce on users and their environment. This is because the designer is in direct contact with users and their everyday contexts, either by observing the activities carried out by the users through contextual inquiry or listening to their stories through storytelling. At that moment the engineer also knows the socio-cultural context of the users, either through the words told (storytelling) or by immersion in the daily work (contextual inquiry), and together with users, think the problems and possible solutions (brainstorm).

\section{2nd stage: Interface Engineering}

The next stage is the interface engineering. This stage has the objective of producing prototypes for the solution envisioned in the previous step. For this, Rosa and Matos suggested the use of braindraw [13]. Braindraw is a technique where users draw the software UI in a collaborative way [9], [13]. The users must be divided into groups of at least 2 and at most 8 participants [9]. Color and graphite pencils, pens, erasers are available and each user receives a sheet of paper. Users should draw their ideas of the software UI and at a set time (e.g. 2 minutes) re-pass the paper to those on the left until each paper was drawn twice by each participant.

Through braindraw, users use their signification systems and previous knowledge to draw an UI considered appropriate for use and containing the problem' solution. Developing it in a collaborative way leads to a fusion of ideas, in which all participants are contributors i.e. the drawing contains characteristics of signification systems of their participants/users [9].

\section{3rd stage: Evaluation}

Finally, the evaluation stage aims to evaluate the produced prototype. At this time, users use their signification systems to interact with the solution that they have designed, with designer mediation. This stage is composed of the think-aloud technique.
For the application of this technique, the designer must design a protocol where the users must follow while interacting with the prototype. During the interaction, the user should speak aloud, as the technique name suggests, about their thoughts, wishes, difficulties, criticisms, suggestions, feelings, and emotions [13]. The interaction should be recorded for further analysis. With think-aloud results, it is possible to identify positive and negative experiences, as well as to identify communication noises that must be repaired before the final version is developed.

\section{A. Researches around SPIDe}

The researches presented by Rosa and Matos [13], and Pita et al. [10] specifically deal with interaction design, where communication between designer and user is restricted to the UI (i.e. to $\mathrm{HCI}$ ). Investigating the communication message composition presented by de Souza [3], Rosa et al. [11], [12] identified that there is a relationship between the message conception and the requirements elicitation process. In this sense, the researchers initiated a project in order to identify if it is possible to elicit requirements through SPIDe.

Initially, a systematic literature review (SLR) was carried out to identify previous research reports that use SPIDe's techniques applied to requirements elicitation [12]. With the SLR, it was identified that only storytelling and brainstorm techniques have already been applied to requirement elicitation; leaving a research gap on the application of other SPIDe techniques, separately or together. In turn, an experimental study was conducted to identify if it is possible to elicit requirements through SPIDe. With the experimental study, the researchers had a positive conclusion [11]. However, this study limits the requirements elicitation, and the researchers did not address how semio-participatory can favor requirements elicitation integrated with interaction design and how the communication happens between designer, engineer, and users. To investigate this gap, in the next section, we present the research methodology.

\section{Methodology}

In order to investigate if the results of SPIDe application satisfies the needs and desires of users, and how communication process occurs during SPIDe application, we carried out an exploratory case study [7], [14]. Data was collected using a logbook [6], a semi-structured interview [7], [14], and Technology Acceptance Model Questionnaire (TAM) [5]. The data analysis was done through the open and axial codings of Grounded Theory [15]. The research was based on the construction of software for a music band in which seven subjects, four users and three specialists, participated. This section details the research planning and execution.

\section{A. Research Planning}

1) Research Questions: To guide the research, the research question is: is SPIDe effective to make communication feasible for requirements elicitation and interaction design? 
2) Case Study: The case study of this research is the software conception for the music band of the Assembly of God church of Ondina, in Salvador/Brazil. The case study was chosen by convenience. The band leader asked the researchers to create a smartphone app to help the band's musicians to perform their activities. The researchers considered that the project was adequate to apply SPIDe and to execute the research, given that in order to construct the demanded solution it was necessary to elicit requirements and also to design the interaction. Complementary, the band members (three subjects) and church pastor ${ }^{3}$, agreed to participate.

3) Participants and Roles: Seven subjects participated in the study $^{4}$, and these were divided into two teams: (a) users and (b) specialists.

All members (three) of the band, Anderson (vocalist and bandleader), Diego (guitarist), and Erick (drummer) and the church pastor (João). The specialists' team was formed by one master and two doctoral students with proven experience in their roles in the software industry. The specialists assumed three roles, namely: SPIDe applicator, requirements engineer, and interaction designer.

4) Data Collection Procedures and Artifacts: Three procedures and artifacts were used to collect data. These procedures are widely used in empirical studies of Software Engineering and HCI. The procedures and artifacts are detailed below.

(a) Logbook [6] - The logbook is an artifact where all participants (specialists and users) can take notes, sketches, calculations and describes feelings, ideas, criticisms, suggestions, observations and other information in support of the research.

(b) Semi-structured interview [7], [14] - The semistructured interview was performed individually, only with the users, after all SPIDe steps were performed, at a moment agreed between users and researchers.

(c) Technology Acceptance Model Questionnaire (TAM) [5] - The TAM aims the identification of the perception about the usefulness and ease of use of SPIDe. The TAM was applied only to the specialists, who answered objective questions on a four-point scale, between totally agreeing and totally disagreeing. Subjective questions were added in order to evaluate negative and positive aspects of SPIDe; aspects of requirements elicitation and interaction design were not contemplated; and the benefit of SPIDe for requirements elicitation and for interaction design in an effective way.

5) Analysis Procedures: The data collected through the procedures and artifacts presented previously was analyzed by means of two phases (of the three) of the Grounded Theory [15]. Since the objective of this research is to construct knowledge about how communication occurs through SPIDe, we only used the open and axial codings. Open coding (1st phase) has the objective of creating codes through abstractions

\footnotetext{
${ }^{3}$ The pastor was invited to participate as he was considered an important stakeholder by the band members and the researchers.

${ }^{4}$ All subjects signed a consent form to participate in the research. The participants also authorized their names publication, except the pastor, who received a fictitious name.
}

related to the collected data. In turn, in axial coding (2nd phase) the codes created in the open coding are analyzed, and relations between them are established, in order to evidence and discover concepts about the research object [15].

\section{B. Execution}

We conducted SPIDe in 4 non-consecutive days of October 2018. In this application, the researchers, in agreement with SPIDe applicator, it was decided not to apply the contextual inquiry, because, in order to apply the technique, the specialists should participate in the band practicing and in church services, which could cause discomfort for both teams, specialists and users.

Initially, it was planned that each stage of the SPIDe would be applied in one day, thus accounting for three days, nonconsecutive. However, on the first day of the research, in which the storytelling and brainstorm would be applied, only Anderson and Diego appeared, and in turn, on the second day, João and Erick attended. Faced with the difference of users who attended the first and second days SPIDe applicator decided to reapply storytelling and brainstorm. This impacted on adding one more day to the SPIDe application. In this days the logbook was also distributed. The third day was dedicated to braindraw and the fourth day to thinking-aloud.

Following the thinking-aloud application, the specialists' team answered the TAM and returned their logbook to the researchers. Subsequently, the researchers contacted the users to conduct the interviews. Nevertheless, the researchers were only able to interview users Anderson, Diego, and Erick, because João did not attend the scheduled meetings. The researchers collected the users' logbook, but due to the lack of contact, it was not possible to collect João's. In turn, Erick did not write in his logbook.

\section{Results AnAlysis}

After data collection, this was analyzed using the open and axial codings of the Grounded Theory [15]. We analyze the data collected through logbook, TAM and semi-structured interviews together, to provide a unified view of the users' team and the specialists' team.

Initially, we showed that the communication between the users was effective since they actively participated in the software conception process because they had "[...] time and freedom to speak". According to Anderson, "the fact that we had plenty of time was what helped us [the users' team] to express everything we had to say". The analysis results indicate that users expressed themselves naturally and "[...] funny way, helping both design and requirements".

Users also pointed out that after applying storytelling they recognized their problem with the help of the SPIDe applicator. While they wrote their ideas in brainstorm's post-its, it was noted by the specialists' team, that users could not describe an exact solution. But when they discussed the ideas together with the specialists' team, the solution was clarified and created together, collaboratively. This expresses that through SPIDe it is possible to build mutual knowledge (between users and 
specialists) on the problem and solution like is provided by the PD ideology [8].

In addition to speaking and writing (which corresponded to the data analysis associated with the context analysis), the users' team could also have signs that they consider appropriate to the UI and also use their previous knowledge. This is demonstrated in braindraw sketchings. Through the interview, Diego pointed out that he had put a button on the UI to have a preview of the song they would play. To do this, he was inspired by the music streaming application Spotify®. Another use of previous knowledge was to have the heart to signify that music is a favorite and stars to rank in notes. Both are signs that are already used in other software, are part of the users' signification systems and are built from their relationship with the culture and society they live.

According to the interaction designer, in interface engineering, the users' creativity "[...] has reached significant levels". According to Diego, "the creation of the application was based on our ideas". But it was not just the sheet and drawing. With sheet rotation for all participants, as indicated by braindraw [9], it was possible for everyone to draw a part of the UI. Users elected "the best [UI] design" democratically, in accordance with what is determined in the PD ideology [8].

After the interface engineering, the specialists turned the drawings into mid-fi prototypes and the users were invited to evaluate it. The specialists have reported that evaluation by think-aloud for some users is not satisfactory. The interaction designer and the SPIDe applicator associated the evaluation with Semiotic Engineering and described signification and communicability problems. In her notes, the SPIDe applicator mentioned that the way in which think-aloud is performed is similar to Communicability Evaluation Method and suggested a change in techniques.

Through the analysis of the collected data, it was still possible to identify that the users consider themselves co-authors of the software. This is because everything has been built collaboratively, between the users and the specialists' team. Thus, according to the research participants, SPIDe reflects what Rosa and Matos [13] thought about the interaction codesign, and goes to a collaborative requirement elicitation. According to the specialists through TAM, the co-authoring provided by SPIDe enables effective communication.

Therefore, SPIDe enables users, their activities, needs, desires, and environments to be (re)cognized (through the use of storytelling and brainstorming) collaboratively; from this, a solution is defined (by means of brainstorm), and also drawn (by braindraw) in a collaborative way. And from the knowledge built in the previous step and with their signification systems, users then interacted with the prototype and evaluated it (through think-aloud).

\section{CONClusions}

This paper presented a semio-participatory methodological process SPIDe; and a case study, with analysis via Grounded Theory, to investigate if the results of SPIDe application sat- isfies the needs and desires of users, and how communication process occurs during SPIDe application.

We concluded that through SPIDe, it is possible for specialists to understand the users desires, needs, and socio-cultural context, given that software conception comes together and initializes from the ideas of its future users. In turn, the problems and solutions that come from the users are clarified as techniques are applied. The specialists' participation is not limited to the observation or techniques application, but these also act in the mediation of communication, to facilitate the mutual construction of knowledge. In this sense, we can conclude that there is evidence in this case study that it is possible to establish effective communication through SPIDe, and achieve satisfactory UI design results, in which users are co-authors. The refinement of SPIDe, as well as the comparison with other processes, are themes for future work.

\section{ACKNOWLEDGMENT}

We would like to thank each of the participants of our research, and LARSyS (UID/EEA/50009/2019). This work is supported by the CAPES-DS (Grant \#1673896); and CAPESPDSE (Grant \#88881.189073/2018-01).

\section{REFERENCES}

[1] P. Bourque and R. E. Fairley, Guide to the Software Engineering - Body of Knowledge. IEEE Computer Society, 2014.

[2] A. B. Cunha, A. G. Canen, and M. A. M, Capretz, "Personalities, cultures and software modeling: Questions, scenarios and research directions," in: Proceedings of ICSE Workshop on Cooperative and Human Aspects on Software Engineering, pp. 23-31, 2009.

[3] C. S. de Souza, The Semiotic Engineering of Human-Computer Interaction. MIT Press, 2005.

[4] V. V. Das, "Involvement of users in software requirement engineering," in: Proceedings - 10th International Conference on Information Technology, pp. 230-233, 2007.

[5] F. Davis, "Perceived usefulness, perceived ease of use, and user acceptance of information technology," MIS Quarterly 13, 3: 319-340, 1989.

[6] H. McAlpine, P. Cash, and B. Hicks, "The role of logbooks as mediators of engineering design work," Design Studies 48: 1-29, 2017.

[7] J. Lazar, J. H. Feng, and H. Hochheiser, Research Methods in HumanComputer Interaction. Cambridge: Morgan Kaufmann, 2017.

[8] R. Luck, "Dialogue in participatory design,” Design Studies 24, 6: pp. 523-535, 2003.

[9] M. J. Muller, J. H. Haslwanter, and T. Dayton, "Participatory Practices in the Software Lifecycle," in Handbook of Human-Computer Interaction (2nd ed.), M. G. Helander, T. K. Landauer and P. V. Prabhu (eds.), Amsterdam: Elsevier, pp. 256-269, 1997

[10] G. L. Pita, D. Zabot, J. C. S. Rosa, and E. Matos, "Adapting the SPIDe to Include Visually Impaired Users in Interaction Design," in Proceedings of the XVI Brazilian Symposium on Human Factors in Computing Systems - IHC 2017, pp. 1-4, 2017.

[11] J. C. S. Rosa, E. Matos, F. S. Silva, and G. J. F. Silva, "Experimentando o SPIDe aplicado à Elicitação de Requisitos," in Proceedings of 21st Workshop on Requirements Engineering, pp. 1-14, 2018.

[12] J. C. S. Rosa, F. S. Silva, G. J. F. Silva, and E. Matos, "Applying SPIDe's Techniques in Requirements Engineering: a systematic review”. Systems and Computing Journal 7, 2: pp. 290-303, 2017.

[13] J. C. S. Rosa and E. Matos, "Semio-Participatory Framework for Interaction Design of Educational Software," in Proceedings of the 15th Brazilian Symposium on Human Factors in Computer Systems - IHC 16, pp. 1-10, 2016.

[14] F. Shull, J. Singer, and D. I. K. Sjberg, Guide to Advanced Empirical Software Engineering. London: Springer, 2008.

[15] A. Strauss and J. Corbin, Basics of Qualitative Research: Techniques and Procedures for Developing Grounded Theory. London: SAGE Publications, 1998 . 